\title{
CADIS has seen the Virgo overdensity and parts of the Monoceros and "Orphan" streams in retrospect (Research Note)
}

\author{
B. Fuchs ${ }^{1}$, S. Phleps ${ }^{2}$, and K. Meisenheimer ${ }^{3}$ \\ 1 Astronomisches Rechen-Institut am Zentrum für Astronomie der Universität Heidelberg, Mönchhofstrasse 12-14, \\ 69120 Heidelberg, Germany \\ e-mail: fuchs@ari.uni-heidelberg.de \\ 2 Max-Planck-Institut für extraterrestrische Physik, Giessenbachstrasse, 85748 Garching, Germany \\ 3 Max-Planck-Institut für Astronomie, Königstuhl 17, 69117 Heidelberg, Germany
}

Received 26 May 2006 / Accepted 13 July 2006

\section{ABSTRACT}

\begin{abstract}
We reanalyze deep star counts in five CADIS fields. The data are presented as vertical density distributions of stars perpendicular to the Galactic plane. In three fields the profiles are consistent with each other, while in two fields significant overdensities of stars are found. The overdensity in one field can be associated with the Virgo overdensity which can be traced right into the disk of the Milky Way. Using this detection we estimate the mass of the Virgo overdensity and show that this is equivalent to the stellar content of a Local Group dwarf spheroidal galaxy. The overdensity in the second field is more difficult to associate with a previously known overdensity. We suggest that it is related both to the Monoceros stream and the recently discovered Orphan stream.
\end{abstract}

Key words. Galaxy: stellar content - Galaxy: structure

In two previous papers (Phleps et al. 2000, 2005) we have studied the distribution of stars in the Milky Way using deep star counts based on Calar Alto Deep Imaging Survey data. For this purpose we analyzed five fields which contained in total 1627 faint stars $(15.5<R<23)$. We do not repeat here any details of our analysis, but give again in Table 1 the coordinates of the fields. At the time of writing the papers we were mainly interested in determining descriptive parameters of the density distributions of the various components of the Milky Way such as the vertical scale heights of the thin and thick disks, respectively, their relative normalization and the parameters of the halo density law. We realized, however, already then that, while the $1 \mathrm{~h}$, $16 \mathrm{~h}$, and $32 \mathrm{~h}$ fields gave very consistent results, the $9 \mathrm{~h}$ and to a lesser degree the $13 \mathrm{~h}$ field lead to discrepant results in the sense that the vertical density distribution of the stars in these fields had a few kpc above the midplane a shallower slope than in the three other fields. With the advent of the Data Release 5 of the Sloan Digital Sky Survey and its subsequent analyses by Jurić et al. (2006) and Belokurov et al. (2006a,b) overdensely populated parts of the density distribution of the stars in the Milky Way have been revealed in such rich detail that we can interpret now in retrospect also the results of the $13 \mathrm{~h}$ field in a consistent way, whereas the nature of the overdensity at $9 \mathrm{~h}$ remains at present less clear. By pure chance two out of the five line-ofsights of the CADIS fields have crossed two separate of such overdense regions! Of course such few line-of-sights did not allow the identification of the excess densities as distinct isolated overdensely populated regions in the Milky Way.

Such features in the density distribution of stars have attracted recently great interest in the literature, because they almost certainly represent debris of satellite galaxies which have fallen into the Milky Way and were then disrupted. Particularly
Table 1. Pointings of the CADIS fields.

\begin{tabular}{rrrrr}
\hline \hline field & RA & \multicolumn{2}{c}{ Dec } & $l$ \\
\multicolumn{5}{c}{ deg } \\
\hline $1 \mathrm{~h}$ & 27 & 2 & 150 & -59 \\
$9 \mathrm{~h}$ & 138 & 46 & 175 & 45 \\
$13 \mathrm{~h}$ & 207 & 6 & 335 & 60 \\
$16 \mathrm{~h}$ & 246 & 56 & 85 & 45 \\
$23 \mathrm{~h}$ & 349 & 12 & 90 & -43 \\
\hline
\end{tabular}

striking are the large filaments like the Sgr stream or the newly discovered "Orphan" stream (Ibata et al. 1997; Majewski et al. 2003; Belokurov et al. 2006a,b; Grillmair 2006) which are interpreted as tidal tails of dwarf galaxies presently in the process of being cannibalized. Obviously such accretion events played an important role in the formation history of the Milky Way.

In this note we present a reanalysis of the CADIS data, because in our view these data still contribute valuable information on the overdensities. In particular we can trace the overdensities right into the disk of the Milky Way, which is not possible with the SDSS data. Moreover, we can provide estimates of the masses of the overdensities.

The CADIS star counts were carried out along the linesof-sight whose coordinates are given in Table 1. In Fig. 1 we show the inferred number densities of stars not as function of heliocentric distance, but as function of the distance from the midplane, i.e. as vertical density profiles perpendicular to the Galactic plane. In this way the five fields are projected onto each other which allows a direct comparison of their density distributions. The distributions of the $1 \mathrm{~h}$ and $23 \mathrm{~h}$ fields have been flipped up. As can be seen from Fig. 1 the distribution of stars 


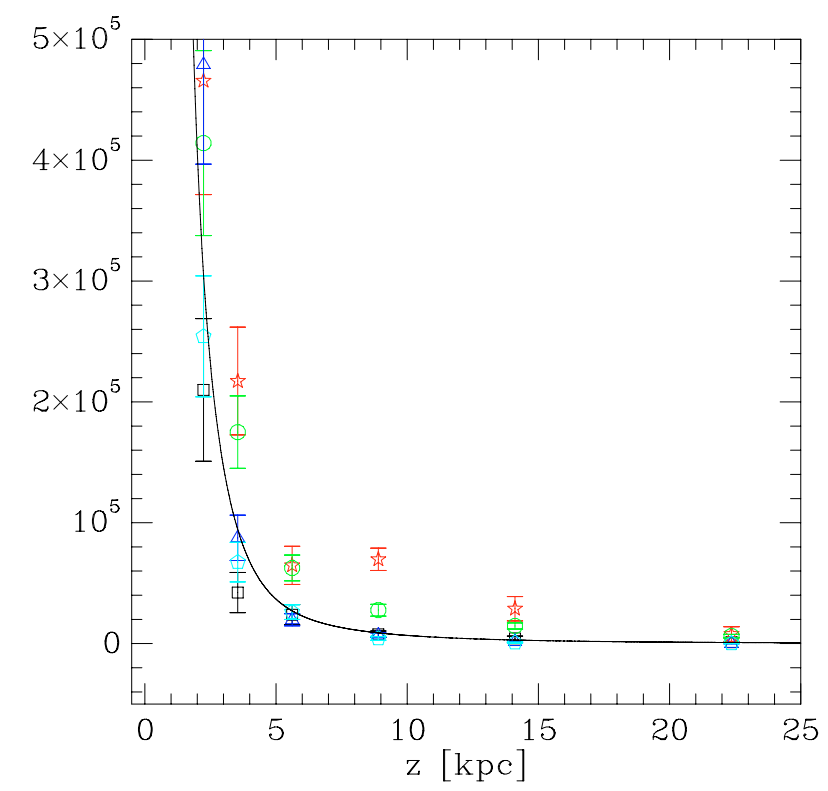

Fig. 1. Number density distribution of stars $\left[* * / \mathrm{kpc}^{3}\right]$ perpendicular to the Galactic plane derived from star counts in CADIS fields. The five fields are coded as: $1 \mathrm{~h}$ (squares), $9 \mathrm{~h}$ (asterisks), $13 \mathrm{~h}$ (circles), $16 \mathrm{~h}$ (triangles), $23 \mathrm{~h}$ (pentagons). Only data above $z=2 \mathrm{kpc}$ are shown (colour coded in the electronic version). The solid line is the vertical density profile at the position of the Sun of a smooth Galaxy model fitted to the data of the $1 \mathrm{~h}, 16 \mathrm{~h}$ and $23 \mathrm{~h}$ fields.

is traced from the outer halo into the disk of the Milky Way. Data below $z=2 \mathrm{kpc}$ are not shown. The density distributions derived from the star counts in the $1 \mathrm{~h}, 16 \mathrm{~h}$, and $23 \mathrm{~h}$ fields, respectively, are within statistical uncertainties consistent with each other. The inclinations of the lines-of-sight of the CADIS fields relative to the vertical axis hardly affect the shape of the density profiles at heights of more then $5 \mathrm{kpc}$ above the midplane. At lower heights part of the scatter among the data shown in Fig. 1 can be ascribed to the varying viewing directions of the CADIS fields. For the present purposes the $1 \mathrm{~h}, 16 \mathrm{~h}$ and $23 \mathrm{~h}$ fields define a vertical reference profile. Having realized this we have repeated the fit of the smooth Galaxy model of Phleps et al. (2005, Eqs. 3 and 5) using only these three fields. As in the previous paper we adopt a vertical scale height of the thin disk of $283 \mathrm{pc}$. The fit to the density distribution in the three reference fields leads to a slightly reduced vertical scale height of the thick disk of $900 \mathrm{pc}$, but to the same normalization of the local density of the thick disk at 4 percent of the total density at the midplane. In the case of a spherical halo model we find an index of the halo density law of $\alpha=3.25 \pm 0.10$ and in the case of a halo flattened as $(c / a)=0.6$ an index of $\alpha=2.69 \pm 0.09$. The density profile perpendicular to the Galactic plane $\left(b=90^{\circ}\right)$ of the smooth Galaxy model, which is shown as a solid line in Fig. 1, is in excellent agreement with the Galaxy model of Jurić et al. (2006).

The $13 \mathrm{~h}$ field shows a statistically significant excess density relative to the reference profile which can be traced from $z=2$ to $14 \mathrm{kpc}$ with the maximal deviation at about $z=4 \mathrm{kpc}$. The heliocentric distances range from 2.3 to $16 \mathrm{kpc}$. The coordinates of this field point towards the fringe of the Virgo overdensity which is discussed in detail by Jurić et al. (2006, cf. their Fig. 24). Indeed, Fig. 1 can be directly compared with Fig. 22 of Jurić et al. (2006), where they delineate the overdensity by subtracting from the density distribution of stars observed in the meridional section of the Milky Way which contains the Virgo

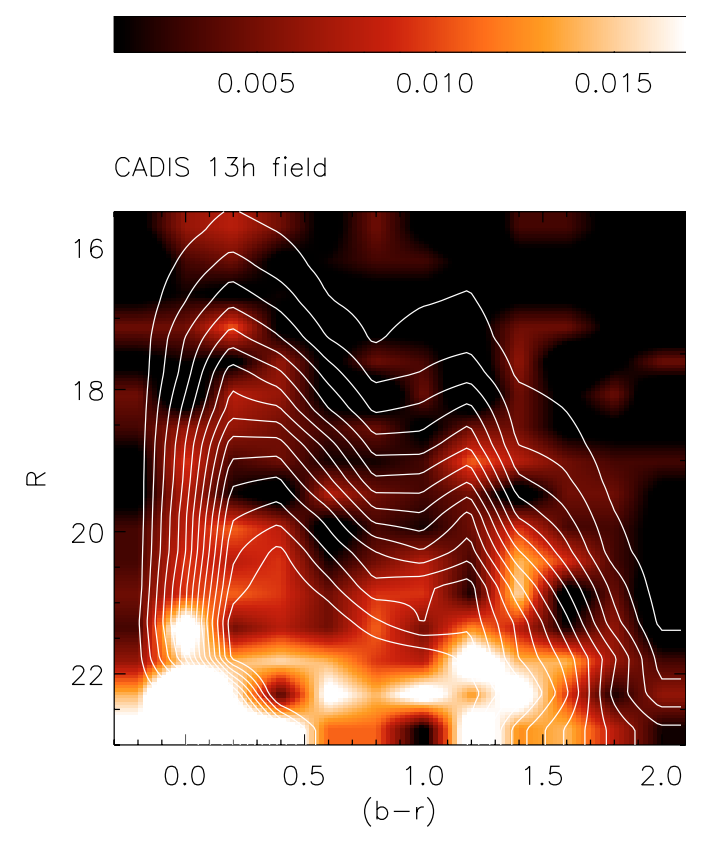

Fig. 2. Hess diagram of the $13 \mathrm{~h}$ field. The observed distribution of stars in the $R-(b-r)$ plane is colour coded according to the number density of stars per bin of size $0.1 \times 0.1$ in magnitude and colour, respectively. The colour table is shown at the top. A smooth model of the Galaxy, which has been fitted to the reference fields, is shown as isodensity contours. The equally spaced contour levels span the same range as the colour table.

overdensity their smooth Galaxy model. Above $z=5 \mathrm{kpc}$, which corresponds to a galactocentric radius of $R=5.5 \mathrm{kpc}$ in the $13 \mathrm{~h}$ field, the vertical profile of the overdensity found in the SDSS DR5 and the CADIS data, respectively, are absolutely consistent with each other. However, in the CADIS data it can be traced right down into the disk of the Milky Way, confirming the supposition of Jurić et al. (2006) that this might be the case.

Moreover, both data sets have been analyzed as Hess diagrams. In their Fig. 24 Jurić et al. (2006) show by subtracting the Hess diagram of a control field from the Hess diagram of the Virgo field that the excess population of stars in the Virgo field is primarily found in the blue branch of the Hess diagram at $(g-r) \approx 0.4$, where halo stars are located. Precisely the same is found in the Hess diagram of the $13 \mathrm{~h}$ field shown in Fig. 2. There is a clear excess in the blue branch of the halo stars at $(b-r) \approx 0.1$ with respect to the smooth Galaxy model, which has been recalculated using the parameters given above. We conclude from this discussion that the excess density in the $13 \mathrm{~h}$ CADIS field can be identified as part of the Virgo overdensity.

Subtracting the reference profile from the observed density profile of the $13 \mathrm{~h}$ field, both now reckoned along the line-ofsight, allows us to determine the mass of the excess population of stars. We find that 276 out of the 517 stars in the $13 \mathrm{~h}$ field seem to belong to the density excess. Phleps et al. (2005) have identified in the CNS4 catalogue (Jahreiß \& Wielen 1997) the analogues of the blue halo stars in the CADIS fields in the local volume (Fuchs \& Jahreiß 1998). They have shown that the extrapolation of the outer halo density law towards the Galactic midplane agrees ideally with the number density of stars with the same colours and absolute magnitudes in the local sample. The average mass of these stars is $0.66 \mathcal{M}_{\odot}$ and the average mass-to-light ratio is $\mathcal{M} / \mathcal{L}_{\mathrm{V}}=2.7 \mathcal{M}_{\odot} / \mathcal{L}_{\mathrm{V} \odot}$. Thus a number density of $10^{5}$ stars per $\mathrm{kpc}^{3}$ corresponds to a mass density of $6.6 \times 10^{-5} \mathcal{M}_{\odot} \mathrm{pc}^{-3}$. We estimate from the area preserving 


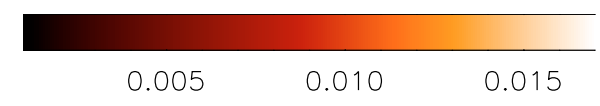

CADIS $9 \mathrm{~h}$ field

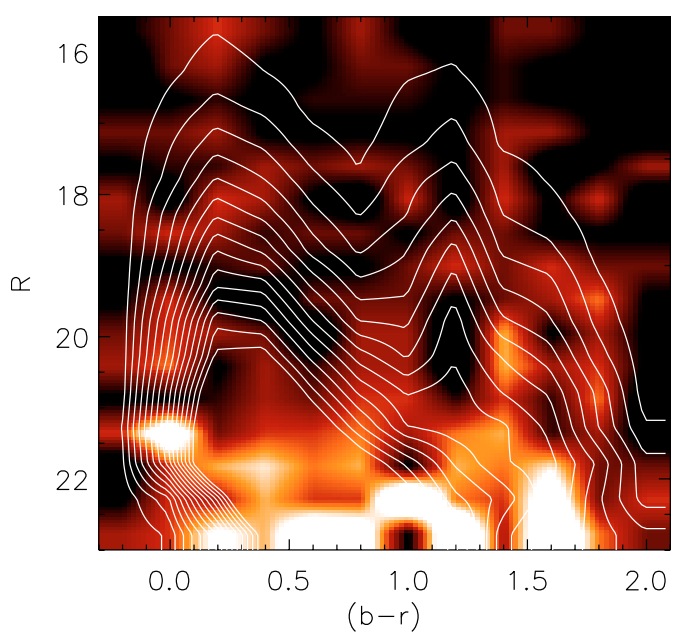

Fig. 3. The same as Fig. 2 but for the 9 h field.

Lambert projection in Fig. 24 of Jurić et al. (2006) that the Virgo overdensity subtends an area of 846 square degrees. One CADIS field has a size of 121 square arcminutes. If the mass of the excess population of stars in the $13 \mathrm{~h}$ field is representative for the rest of the Virgo overdensity the latter contains a mass of $4.6 \times 10^{6} \mathcal{M}_{\odot}$. This is in our view a clear indication that the Virgo overdensity is the relic of a shredded dwarf galaxy. Adopting the mass-to-light ratio of $2.7 \mathcal{M}_{\odot} / \mathcal{L}_{\mathrm{V} \odot}$ found above the stars in the Virgo overdensity would have a total luminosity of $1.7 \times 10^{6} \mathcal{L}_{\mathrm{V} \odot}$ or an absolute magnitude of $\mathrm{M}_{\mathrm{V}}=-11 \mathrm{mag}$, which is quite typical for Local Group dwarf spheroidal galaxies. Also the inferred mass-to-light ratio is typical for the stellar populations of dwarf spheroidals (Mateo 1998).

The $9 \mathrm{~h}$ field shows at distances between $z=2$ and $15 \mathrm{kpc}$ above the midplane an overdensity relative to the reference profile, which is even more pronounced than that in the $13 \mathrm{~h}$ field. The maximal deviation is found around $z=9 \mathrm{kpc}$. The corresponding heliocentric distances range from 3 to $21 \mathrm{kpc}$. However, this overdensity is more difficult to associate confidently with one of the overdensities seen in the SDSS data (Juric et al. 2006; Belokurov et al. 2006a,b). We note that most of the density excess in the Hess diagram of the $9 \mathrm{~h}$ field shown in Fig. 3 is found in the colour range $(b-r) \approx 0.5-1$ and falls right into the gap between the two branches of the disk and halo stars, respectively. Judging from the Hess diagrams presented by Juric et al. (2006) for the Virgo field and its control field stars of such intermediate colour have been eliminated by the colour cut $(g-r)<0.4$ from the sample of Belokurov et al. (2006a). In Fig. 1 of Belokurov (2006b) there is also the distribution of stars with colours $0.4<(g-r) \leq 0.6$ shown and we find the $9 \mathrm{~h}$ field at the eastern fringe of the northern part of the Monoceros stream (Newberg et al. 2002) which seems to be in this colour range much more extended than in the bluer $(g-r)<0.4$ colours (Belokurov et al. 2006a). Similarly the Monoceros stream is seen in the data of Juric et al. (2006, their Fig. 9) mainly at slightly lower galactic latitudes than that of the $9 \mathrm{~h}$ field. Its line-of-sight passes though through the inner fringe of the overdensity in anticenter direction in the panels of Fig. 9 which show the density distribution of stars with colours $0.1<(r-i) \leq 0.15$ at heights of 4 and $5 \mathrm{kpc}$ above the midplane. Jurić et al. 2006) do study the distribution of redder stars, but cannot trace it beyond a few kpc from the Sun. Peñarrubia et al. (2005) have modelled the Monoceros stream by numerical simulations as a tidal stream. Their simulations show that part of the stream might be very well seen in the direction of the $9 \mathrm{~h}$ field and at the heliocentric distances of the overdensity in the $9 \mathrm{~h}$ field. Thus this overdensity may be tentatively associated with the Monoceros stream. However, there is a further aspect of the interpretation of the overdensity in the $9 \mathrm{~h}$ field. The $9 \mathrm{~h}$ field is positioned exactly on the galactocentric great circle on which the Orphan stream lies and which passes also through the high velocity cloud complex A (Belokurov et al. 2006a,b; Wakker 2001). The position is roughly in the middle between the northern tip of the Orphan stream and Complex A. Belokurov et al. (2006b) have determined a heliocentric distance of the northern tip of the Orphan stream of $35 \pm 10 \mathrm{kpc}$, whereas the distance to Complex $\mathrm{A}$ is $10.1 \pm 0.9 \mathrm{kpc}$. This discrepancy can be resolved in a natural way if Complex A is on a different wrap around the Galactic center as the Orphan stream. The density profile of the $9 \mathrm{~h}$ field gives the impression that there is an extra "hump" in the density excess of stars relative to the reference fields which is centered around $z \approx 9 \mathrm{kpc}$ or a heliocentric distance of $13 \mathrm{kpc}$. It is tempting to speculate that the "hump stars" are associated with the second wrap of the Orphan stream. The width of the Orphan stream is estimated to be only a few $100 \mathrm{pc}$ (Belokurov et al. 2006b). That would be consistent with a narrow feature in the density profile of the overdensity. The fairly long elongated overdensity in the density profile of the $9 \mathrm{~h}$ field must be then still ascribed to the Monoceros stream.

In summary, we conclude that the Virgo overdensity has almost certainly been seen in the $13 \mathrm{~h}$ CADIS field, although it was not recognized previously by us as such. We have found in the $9 \mathrm{~h}$ field another overdensity which is as significant as the Virgo overdensity in the $13 \mathrm{~h}$ field which we tentatively attribute to the Monoceros and Orphan streams. Interestingly both features could be traced to distances less than $3 \mathrm{kpc}$ from the Sun. If they are related to any of the star streams identified as fine structure in the phase space distribution function of stars in the solar neighbourhood (Helmi et al. 1999; Chiba \& Beers 2001; Navarro et al. 2004; Helmi et al. 2006; Arifyanto \& Fuchs 2006) is at present unclear.

Acknowledgements. We thank Vasily Belokurov, Wyn Evans and Hans-Walter Rix for very helpful discussions.

\section{References}

Arifyanto, M. I., \& Fuchs, B. 2006, A\&A, 449, 533

Belokurov, V., Zucker, D. B., Evans, N. W., et al. 2006a, ApJ, 642, L137

Belokurov, V., Yanny, B., Zucker, D., et al. 2006b, ApJ, submitted

Chiba, M., \& Beers, T. 2001, ApJ, 549, 325

Fuchs, B., \& Jahreiß, H., 1998, A\&A, 329, 81

Grillmair, C. J. 2006, ApJ, in press

Helmi, A., White, S. D. M, de Zeeuw, P. T., \& Zhao, H. 1999, Nature, 402, 53

Helmi, A., Navarro, J. F., Nordström, B., et al. 2006, MNRAS, 365, 1309

Ibata, R. A., Wyse, R. F. G., Gilmore, G., et al. 1997, AJ, 113, 634

Jahreiß, H., Wielen, R., 1997, in HIPPARCOS '97, ed. B. Battrick, M. A. C. Perryman and P. L. Bernacca, ESA SP-402, 675

Jurić, M., Ivezić, Z, Brooks, A., et al. 2006, ApJ, in press

Majewski, S. R., Skrutskie, M. F., Weinberg, M. D., \& Ostheimer, J. C. 2003, ApJ, 599, 1082

Mateo, M. 1998, ARAA, 36, 435

Navarro, J. F., Helmi, A., Freeman, K. C. 2004, ApJ, 601, L43

Newberg, H. J., Yanny, B., Rockosi, C. et al. 2002, ApJ, 569, 245

Peñarrubia, J., Martínez-Delgago, D., Rix, H.-W., et al. 2005, ApJ, 626, 128

Phleps, S., Meisenheimer, K., Fuchs, B., \& Wolf, C. 2000, A\&A, 356, 108

Phleps, S., Drepper, S., Meisenheimer, K., \& Fuchs, B. 2005, A\&A, 443, 929

Wakker, B. 2001, ApJS, 136,463 